DOI: https://doi.org/10.46296/yc.v5i9edespsoct.0113

\title{
REDES SOCIALES, CREATIVIDAD Y APRENDIZAJE EN LÍNEA EN ESTUDIANTES DE BACHILLERATO
}

\section{SOCIAL NETWORKS, CREATIVITY AND ONLINE LEARNING IN HIGH SCHOOL STUDENTS}

\author{
Ruiz-Loor Jenny Lourdes ${ }^{1}$; Gallegos-Macías Marcos Ramón ${ }^{2}$ \\ ${ }^{1}$ Universidad San Gregorio de Portoviejo USGP. Portoviejo Ecuador. Correo: \\ e.jlruiz@sangregorio.edu.ec. ORCID ID: https://orcid.org/0000-0003-0516-5907 \\ 2 Universidad San Gregorio de Portoviejo USGP. Portoviejo Ecuador. Correo: \\ mgallegos@sangregorio.edu.ec. ORCID ID: https://orcid.org/0000-0002-3651-034x
}

\section{Resumen}

La presente investigación radica en el impacto positivo que tiene la implementación de las redes sociales en el aprendizaje en línea lo que nos invita a inculcar su adecuado uso, ya que son cada vez más utilizadas por la riqueza y opciones que brindan para el diseño y desarrollo de actividades de aprendizaje, sobre todo aquellas que se relacionan con el aprendizaje colaborativo y cooperativo. Para este estudio se planteó como objetivo valorar la calidad del uso de las redes sociales que utilizan los estudiantes de primero de bachillerato. Se fundamentó en referencias bibliográficas actualizadas y confiables que caracterizaron las variables de estudio. El diseño metodológico tuvo un enfoque cualitativo-cuantitativo y bibliográfico. Se aplicó encuestas a docentes y estudiantes. Los hallazgos de la investigación determinaron que el uso de las redes sociales por parte de los estudiantes está relacionado a acciones informales, recreativas, comunicativas entre sus pares, mas no con un fin pedagógico ni de aprendizaje. aun siendo estas un medio de comunicación, de información, de formación actual e interactivo tanto de enseñanza como de aprendizaje, ayudándoles a profesores como a estudiantes, a familiarizarse con herramientas y aplicaciones.

Palabras claves: aprendizaje en línea; comunicación; creatividad; enseñanza; redes sociales.

\begin{abstract}
This research is based on the positive impact that the implementation of social networks has on online learning, which invites us to inculcate their proper use, since they are increasingly used due to the wealth and options they provide for design and development. of learning activities, especially those related to collaborative and cooperative learning. The objective of this study was to assess the quality of the use of social networks used by first year high school students. It was based on updated and reliable bibliographic references that characterized the study variables. The methodological design had a qualitative-quantitative and bibliographic approach. Surveys were applied to teachers and students. The research findings determined that the use of social networks by students is related to informal, recreational, communicative actions among their peers, but not with a pedagogical or learning purpose. even though these are a means of communication, information, current and interactive training for both teaching and learning, helping teachers and students to become familiar with tools and applications.
\end{abstract}

Keywords: online learning; communication; creativity; teaching; social media.

Información del manuscrito:

Fecha de recepción: 22 de julio de 2021.

Fecha de aceptación: 24 de septiembre de 2021.

Fecha de publicación: 01 de octubre de 2021. 


\section{Introducción}

Tomando como punto de partida la emergencia sanitaria concedió existencia social a la posibilidad de estudiar utilizando los entornos de educación en línea. De pronto, y literalmente de la noche a la mañana, el Gobierno presentó un programa para que los alumnos eximidos de asistir a la escuela, pudieran utilizar la red para "aprender". Frente a la crisis, las instituciones empezaron a reaccionar con los recursos que tenían disponibles, y al hablar de recursos no me refiero a las máquinas, sino a la capacidad institucional instalada para poder pensar cómo crear espacios de aprendizaje en la red. Muchas instituciones educativas tuvieron que montar la presencia institucional en la Red y cómo utilizarla con propósitos educativos.

Consecuentemente esto permitió profundizar sobre el tema en función de hacer énfasis en justificar y argumentar sobre el uso de las redes sociales y la creatividad del aprendizaje en línea de los estudiantes, de ahí la relevancia de evaluar el impacto que éstas tienen como facilitadoras del aprendizaje. Las tecnologías de la información y la comunicación (Tics) son hoy una herramienta necesaria en muchos ámbitos y son fundamentales como complemento de una educación de calidad en todos los niveles.

Las redes sociales en Internet se han convertido en una de las formas de comunicación más usadas por niños y jóvenes. A pesar de las numerosas oportunidades que nos ofrecen las nuevas tecnologías, es necesario tener presente también algunos obstáculos y/o desafíos con los que nos podemos encontrar y a los que debemos hacer frente. El estudio Virtual Models of European Universities, realizado por la asesoría danesa Ramboll Managenent para la Comisión Europea, entre los años 2002 y 2003, recoge algunos de ellos: a) Para muchas universidades uno de los principales desafíos es adaptar las nuevas tecnologías al ámbito de la docencia; b) Falta de conocimientos. La mayoría de los docentes afirman carecer de conocimientos para poder usar las nuevas tecnologías; c) Escasez de recursos relacionados con las nuevas tecnologías. Todavía la 
elaboración de materiales resulta demasiado cara. (Muñoz, Fragueiro, \& Ayuso, 2017, pág. 5) El uso de las Tics a través de la implementación de las redes sociales en la enseñanza en el siglo XXI es fundamental más aun en estos momentos de pandemia mundial donde la educación es virtual, por tanto, esta y las investigaciones posteriores constituyen una pauta innovadora para fortificar este trabajo investigativo.

Este estudio de (Acosta \& Apolo, 2018, pág. 1) se estableció que un $80 \%$ de los jóvenes usuarios de medios han utilizado las redes sociales con fines educativos, aspecto que ha posibilitado llegar a la conclusión que el uso de tecnologías digitales no sólo está contribuyendo a fomentar aspectos como la comunicación y el entretenimiento, sino también incorporar aprendizajes invisibles a sus procesos cognitivos.

El uso de las redes sociales para influir en las actitudes ambientales de los profesionales en formación en una universidad pública domiciliada en Manabí, Ecuador. Se aplican técnicas de investigación cualitativa para colectar las voces de los informantes, las que son analizadas desde el enfoque sociocrítico. Los informantes son 40 estudiantes de una universidad pública, quienes participan en un proyecto de investigación acción para estudiar sus actitudes ambientales y comportamiento resiliente. Se articulan foros mediante Facebook y YouTube; y se ejecutan actividades para la exploración del entorno por medio de la red social WhatsApp. Como resultado se identificaron acciones dirigidas a la reducción del consumo de electricidad, papel y agua potable en el entorno universitario. Se concluye que, las redes sociales aportan a los procesos de innovación de la formación profesional ofreciendo espacios de aprendizaje que promueven la participación, reflexión y proponen acciones resilientes al cambio climático en la universidad del siglo XXI (Villafuerte \& Rodríguez, 2019, pág. 4). Las redes sociales constituyeron un desafío importante para los docentes, principalmente en la educación superior debido a que son el principal medio de interacción debido a su inmediatez y facilidad de acceso. Estas herramientas tecnológicas se han implementado de una manera 
inmediata en el ámbito educativo y pueden ser enfocadas a varias actividades académicas, con el fin de que complementen la enseñanzaaprendizaje y contribuyan, por ende, a la construcción del conocimiento.

Para este estudio se planteó como objetivo valorar la calidad del uso de las redes sociales que utilizan los estudiantes de primero de bachillerato de la Unidad Educativa Francisco Pacheco. La investigación fue de carácter fundamental y necesaria dado que los estudiantes con frecuencia hicieron uso de las redes sociales, sin embargo, estos fueron con fines poco productivos lo cual influyo negativamente en su aprendizaje.

La relevancia de este proyecto investigativo radico en el impacto positivo ante la sociedad y la comunidad educativa dado que, esta metodología invita a que la comunidad continúe sumándose a la loable labor de inculcar el adecuado uso de las redes sociales en el proceso de enseñanza-aprendizaje.

Las redes sociales con fines educativos son cada vez más utilizadas por la riqueza y opciones que brindan para el diseño y desarrollo de actividades de aprendizaje, sobre todo aquellas que se relacionan con el aprendizaje colaborativo y cooperativo. La incorporación de estas redes como estrategia educativa nos obliga a reconocer que la comunicación y la interacción se desarrollan de distinta manera, por lo que es conveniente tomar en cuenta las dimensiones, características y comunidades que forman una red.

La revisión de algunos estudios sobre el uso académico de las redes sociales permite identificar que se requiere trabajar en planteamientos didácticos con objetivos que orienten la comunicación, desarrollo y exhibición de las producciones de profesores y estudiantes, para obtener el mayor provecho de lo que hasta el momento se vislumbra como potencialidad en el campo educativo. (Pérez, Ortíz, \& Flores, 2016, pág. 18).

Cada vez se expande con mayor intensidad una vida digital que impulsa a las organizaciones a sumergirse en las nuevas formas de comunicación por medio de Internet. Las organizaciones deben adaptar su cultura y metodología para sacar partido al gran potencial que ofrecen 
las redes sociales. Este trabajo muestra cómo existen diferentes iniciativas en distintos ámbitos en cuanto al uso de las redes sociales. Además, facilita la coordinación y trabajo de diversos grupos de aprendizaje (clase, asignatura, grupo de alumnos de una asignatura, etc.) mediante la creación de los grupos apropiados. (Silva \& Cadena, 2018, pág. 4).

A continuación, abordamos las redes sociales desde su definición, beneficios, uso, características y hasta como utilizarla con creatividad en el aprendizaje en línea.

Redes sociales en la educación

Son redes que fomentan comunidades virtuales, cuyos intereses les brinda la capacidad de construir situaciones de aprendizajes significativos. (Freire, Díaz, \& Vera, 2018, pág. 3) Las redes sociales contribuyen en el aprendizaje de los estudiantes dado que, aunque se la realiza en un ambiente informal se puede difundir contenido didáctico e interactivo fomentando así el interés por parte de los estudiantes, tanto para generar contenido o participar en las diversas actividades planteadas. La aplicación de esta metodología requiere la participación constante tanto del docente como del estudiante para fomentar el aprendizaje autónomo.

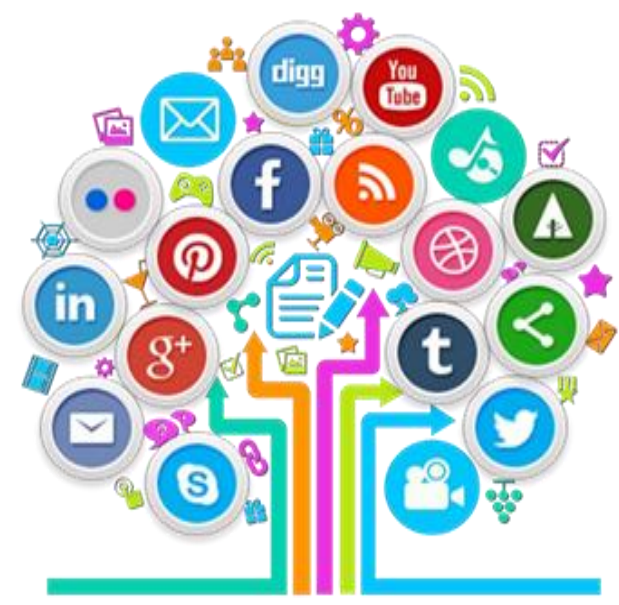

Fuente: Tics y derecho a la libertad.

\section{Beneficios del uso de las redes sociales en el aprendizaje}

El uso de las redes sociales para el aprendizaje proporciona grandes beneficios para la comunidad educativa según la (Organización del Bachillerato Internacional, 2020, pág. 12) son los siguientes: a) facilitación de materiales multimedia al alumno o participante; b) conectar a los alumnos o participantes con otros; c) combinación de contenido visual, seminarios web, discusiones en línea y chats; d) crear exposiciones virtuales con texto e imágenes; e) constituir un espacio 
virtual eficaz para compartir y aprender. Las redes sociales son medios de comunicación para la respectiva difusión de contenido científico, orientaciones metodológicas, asignación de tareas y para fortalecer el desarrollo de destrezas, capacidades y habilidades de los estudiantes mediante la participación e interacción en la comunidad en línea.

Uso adecuado de las redes sociales en el aprendizaje en línea

El uso adecuado de las redes sociales en el aprendizaje en línea está a cargo de los padres 0 representantes debido a el confinamiento, por tanto, el (Ministerio de Educación, 2020, pág. 28) dice que es indispensable que los miembros de la familia puedan acompañar a los estudiantes en el desarrollo de las tareas en casa mientras dure la emergencia sanitaria.

La familia debe garantizar el lugar, tiempo y uso adecuado a la plataforma educativa virtual, pues también tenemos la oportunidad de desarrollar competencias digitales en niños y adolescentes. En actualidad en el aprendizaje en línea es de vital importancia por ende es fundamental plantear actividades interactivas tales como: producción de contenido mediante redacción colaborativa; cuestionarios y juegos interactivos; publicación de blogs creación de historias publicación de foros video llamadas en tiempo real y otros. También es imprescindible acatar las siguientes sugerencias establecidas en el Plan Educativo Aprendamos Juntos en casa respecto al tiempo de pantalla en niños y adolescentes: de 7 - 12 años una hora; de 12 - 15 años una hora y media; más de 16 años dos horas, todos los niños y adolescentes deben contar con la compañía de un adulto durante el tiempo de conexión.

\section{Característica de la creatividad del} aprendizaje en línea

Según (Avid \& Bermeo, 2018, pág. 44) las características del aprendizaje en línea son las siguientes: a) Flexibilidad: Permite adaptarse a la individualidad del que aprende, cada quien aprende en sentido de su alcance; b) Originalidad: Usa su creatividad para proponer resultados y respuestas; c) Abstracción: El aprendizaje creativo le permite al aprendiz tomar 
información de cualquier naturaleza

y adaptarla al proceso; d)

Introspección: El sujeto que aprende hace una reflexión, individual para luego por medio de la creatividad dar a conocer sus logros de aprendizaje. Las redes sociales son recursos de internet, comunicacionales que hacen posible el constante contacto entre uno o más seres a pesar de las distancias geográficas existentes. Por tanto, la implementación del aprendizaje creativo y a través del uso de las redes sociales es de vital importancia para el desarrollo de los modelos de aprendizaje, dado que, de esta mediante la aplicación de esta estrategia se despertará el interés de los estudiantes y esto facilitará la comprensión de diversos contenidos con la difusión y observación de imágenes y videos didácticos e ilustrativos.

\section{Cómo se implementa la creatividad del aprendizaje en línea}

El aprendizaje autónomo es un aprendizaje transcendental en el que las personas toman decisiones importantes sobre su propio aprendizaje, lo cual les permite autodirigirlo en función de unas necesidades, metas o propósitos, acciones, tiempos y de acuerdo con los recursos que disponen. El aprendizaje autónomo facilita que las personas aprendan a aprender a través del ejercicio y desarrollo de competencias 0 habilidades cognitivas, afectivas e interactivas. (Kyriacou, Negrete, \& René, 2016, pág. 7) Los docentes tomen la iniciativa de implementar el uso de redes sociales como herramienta didáctica ya que los alumnos de hoy es una generación nacida junto con las nuevas tecnologías y son adictivamente atraídos por los dispositivos electrónicos. Por tal motivo mediante el aprendizaje colaborativo y autónomo en las redes sociales se deberá aprovechar este potencial para aplicar las estrategias que contribuyan con el desarrollo del aprendizaje colaborativo por medio de la participación en foros, debates y otras actividades.

Por otra parte, para el desarrollo del aprendizaje autónomo es importante promover la creatividad a través de la elaboración de infografías, videos y otros recursos. 


\section{Métodos}

La investigación tuvo un enfoque cualitativo-cuantitativo ya que se usó la recolección de datos para probar hipótesis, con base en la medición numérica y el análisis estadístico, para establecer patrones de comportamiento y probar teorías, es decir que se abordó el tema de estudio desde los aspectos cuantitativos y medibles; a fin de identificar hallazgos de importancia que sirvan de base a la investigación.

De la misma manera, se aplicó la investigación bibliográfica, mediante el análisis de textos especializados, respecto al objeto en estudio considerando aquellos producidos durante la última década para tener la información más actualizada.

Se implementó una investigación analítica y deductiva que nos ayudó a cómo entender y así poder observar las causas, la naturaleza y los efectos que puede tener esta investigación, estos métodos nos permitieron conocer más el objetivo de estudio que se pueda presentar para así explicar y comprender cada uno de los comportamientos y establecer nuevas teorías.
Es fundamental saber qué respuesta nos pueden dar cada una de las teorías, leyes he hipótesis que se vallan a implementar y tener pautas para poder relacionarla con las variables que tengamos y poder ir más lejos intentando descubrir todos los mecanismos que se encontraron en la investigación y poder resolver cada uno de ellos.

Las técnicas de investigación que se utilizaron fueron, mediante la recolección de datos por medio de encuestas a 19 docentes de bachillerato y 31 estudiantes de Primero de bachillerato para evidenciar los resultados de la investigación.

La información primaria fue proporcionada por los docentes y estudiantes de la Unidad Educativa Francisco Pacheco, como fuente secundaria se contó con repositorios de universidades, libros digitales, artículos científicos. Las técnicas de procesamiento y análisis de los resultados, que se utilizaron fueron métodos estadísticos, mediante la recolección de datos por medio de encuestas con preguntas de base estructurada a docentes y estudiantes realizadas en formularios de Google forms. 


\section{Resultados}

En relación con los datos obtenidos de los instrumentos que se aplicaron en la investigación se presenta los resultados de la encuesta digital aplicada a los estudiantes, que permitió evidenciar que el $41.94 \%$ de los estudiantes casi nunca utilizan las redes sociales para mantener interactividad con sus docentes, según lo menciona (Artero, 2011, pág. 25) "El profesor deja de ser la fuente de transmisión de saber para su alumnado, puesto que toda la magnitud que se quiera de conocimiento reside en la Red, y debe ser consciente de ello y legitimar su posición en el aula como guía, tutor y mediador en el aprendizaje". El uso de las redes sociales en el ámbito educativo ayuda a fomentar el valor de compartir y colaborar, de esta forma no sólo el docente transmite conocimientos, sino que favorece la colaboración entre las personas. Permitiendo que el docente les enseñe a sus estudiantes a aprender por sí mismos. Hoy en día el formador, a la vez que enseñar debe actuar de guía para que los alumnos descubran por sí solos nuevas fuentes de aprendizaje que nos brinda el internet.

Tabla 1. ¿Con que frecuencia ha utilizado las redes sociales para mantener interactividad con sus docentes?

\begin{tabular}{ccr}
\hline № & Alternativas & Porcentaje \\
\hline 1 & Casi todos los días & $35,48 \%$ \\
2 & Todos los días & $9,68 \%$ \\
3 & Nunca & $12,90 \%$ \\
4 & Casi nunca & $41,94 \%$ \\
\hline & TOTAL & $\mathbf{1 0 0 , 0 0 \%}$ \\
\hline
\end{tabular}

Según los resultados obtenidos en la encuesta aplicada el $32.26 \%$ de los estudiantes utilizan las redes sociales para enviar mensajes y comunicarse con sus amigos, familiares $u$ otras personas a las que ya conocen, según como menciona
(Freire, Cepeda, \& y Santiago, 2017, pág. 126) El inicio del siglo XXI ha venido marcado por una verdadera revolución informativa y colaborativa apoyada en la tecnología y las telecomunicaciones. Las redes sociales se revelan como el gran 
cambio social, económico y educativo de dicha revolución, formando, cada vez más, parte activa de nuestras vidas. Siendo este proceso difícil de conferir en la medida que desconocemos tanto su potencial como los riesgos a los que nos podemos ver sometidos. El uso de las redes sociales en el ámbito educativo juega un papel muy importante porque les permite a los estudiantes a sentirse más cómodos fomentando confianza. Además, las redes sociales pueden emplearse para el trabajo en equipo, así como para lograr una comunicación más eficiente entre familias, docentes y la propia institución educativa.

Tabla 2. ¿De las siguientes actividades que realiza en las redes sociales, por favor indique cuales realiza?

\begin{tabular}{ccc}
\hline № & Alternativas & Porcentaje \\
\hline 1 & Enviar mensajes y comunicarse con sus amigos, & $32,26 \%$ \\
& familiares u otras personas a las que ya conoce. & \\
2 & Conocer gente nueva o hacer nuevos amigos. & $19,35 \%$ \\
3 & Intercambiar fotos, videos, música, etc. & $12,90 \%$ \\
4 & Participar en debates o discusiones sobres los temas & $9,68 \%$ \\
& Simple interés. & $19,35 \%$ \\
6 & Realizar trabajos en grupos con sus compañeros de & $6,45 \%$ \\
& colegio. & $\mathbf{1 0 0 , 0 0 \%}$ \\
\hline
\end{tabular}

De acuerdo con los resultados que se obtuvo de la encuesta digital aplicada a los docentes, el $52.63 \%$ consideran que es absolutamente necesario el uso de las redes sociales como herramienta pedagógica, según como lo menciona (Meso, 2010, pág. 25) Las Redes Sociales es otro tipo de situaciones de aprendizaje, el esfuerzo del docente se va a centrar en ayudar al educando a desarrollar las competencias requeridas como parte de su nivel de formación en que se encuentra nuevos esquemas de enseñanza, lo cual lo convierte en un guía del proceso de enseñanzaaprendizaje. Por su parte, el estudiante se vuelve un ser más activo, independiente, autónomo y responsable de su propio proceso de aprender, que construye sus propios conocimientos, el profesor ahora tiene la labor de ser un facilitador del 
conocimiento que lo ayuda a Nunca antes ha sido tan fácil para las aprender. mentes jóvenes crear una imagen

Las redes sociales como digital de sus acciones a través de un herramienta pedagógica les brindan a los estudiantes tener libertad de actuar simplemente sentándose frente a una computadora 0 medio tan espontáneo. capacidad de análisis, criticidad y reflexión, con una metodología basada en los soportes tecnológicos.

Smartphone.

Tabla 3. ¿Qué opina usted del uso de las redes sociales como una herramienta pedagógica que promueve el aprendizaje en línea?

\begin{tabular}{ccc}
\hline № & Alternativas & Porcentaje \\
\hline 1 & Absolutamente innecesario & $5,26 \%$ \\
2 & Inncesario & $0,00 \%$ \\
3 & Ni innecesario ni necesario & $0,00 \%$ \\
4 & Mayormente necesario & $42,11 \%$ \\
5 & Absolutamente necesario & $52,63 \%$ \\
\hline & TOTAL & $\mathbf{1 0 0 , 0 0 \%}$
\end{tabular}

Con respecto a estos resultados, el $25.81 \%$ de los estudiantes utilizan las redes sociales para realizar combinación de contenido visual, discusiones en línea y chats, según como lo menciona (Goméz, 2012, pág. 9) Las redes favorecen la publicación de información, el aprendizaje autónomo, el trabajo en equipo, la comunicación, la realimentación, el acceso a otras redes afines y el contacto con otros expertos, entre otros elementos. Las redes sociales aplicadas en la educación permiten abrirles nuevas oportunidades de aprendizaje a los estudiantes $\mathrm{y}$, por medio de la innovación didáctica favorecer un aprendizaje colaborativo y el diálogo entre compañeros. 
Figura 1. ¿Seleccione los beneficios que pueden tener las redes sociales orientadas a la educación?

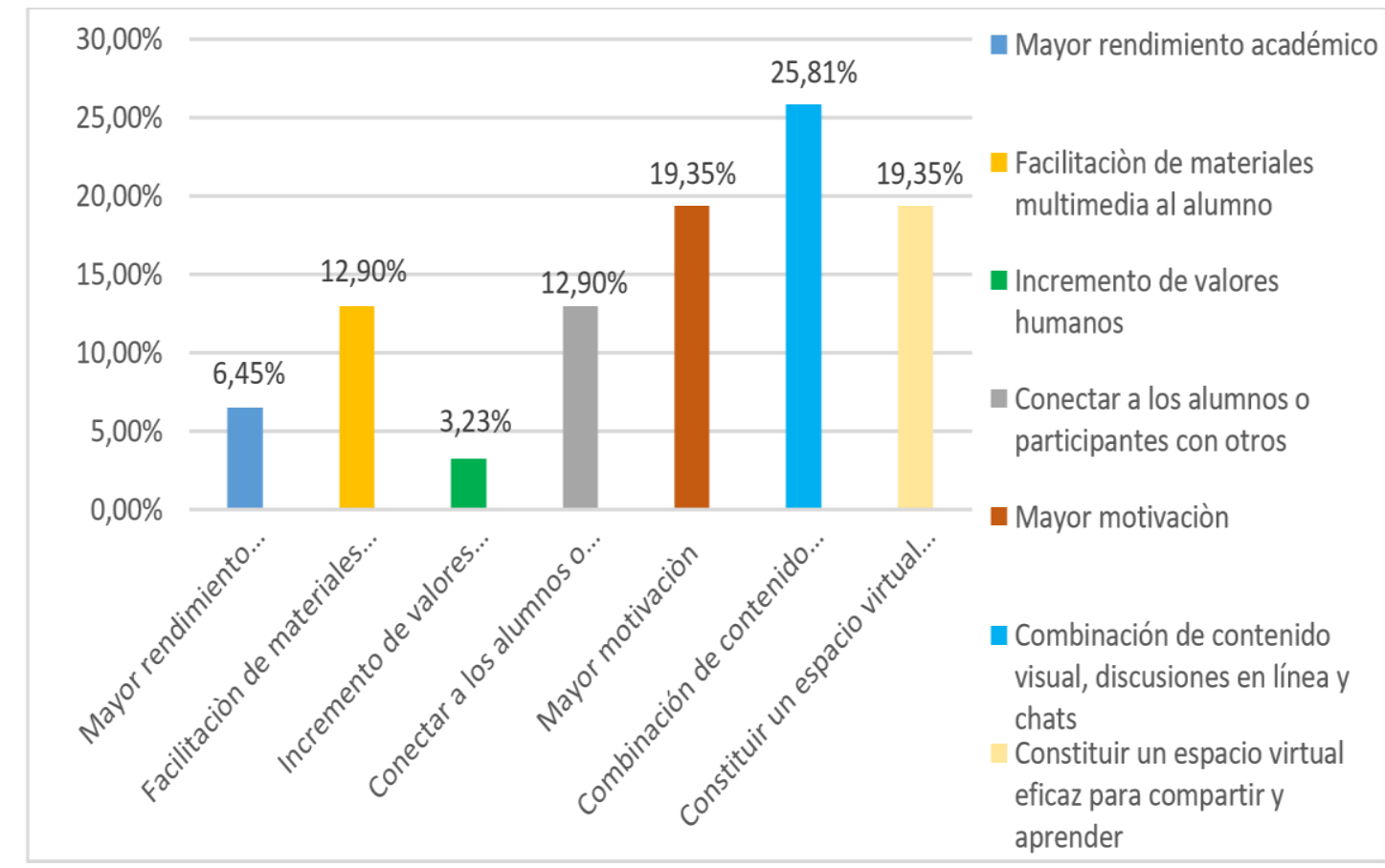

También se pudo evidenciar con los datos obtenidos de esta investigación que el $47.37 \%$ de los docentes consideran estar de acuerdo en que el uso de las redes sociales aporta a la creatividad del aprendizaje en línea, según como lo menciona (Bohorquéz \& Rodríguez, 2017, pág. 6) Hoy en día las redes sociales permiten establecer relaciones sin importar el espacio físico con una gran facilidad de acceso y comunicación, bridando espacios de interacción y desenvolvimiento para jóvenes, ya que a través de los dispositivos electrónicos pueden tener fluidez en la comunicación. Las redes sociales en Internet se han convertido en una de las formas de comunicación más usadas por niños y jóvenes. pueden ser una herramienta de gran valor en el ámbito educativo, pues se convierte en una nueva forma de enseñar y también de aprender, en la que el alumno podrá desarrollar un importante trabajo cooperativo crear un ambiente participativo se convierten en nuevas oportunidades de crecimiento a nivel personal y académico. Es un espacio ideal para compartir conocimientos que resulten atractivos y motivadores para los estudiantes. 
Figura 2. ¿Según su opinión el uso de las redes sociales aporta a la creatividad del aprendizaje en línea?

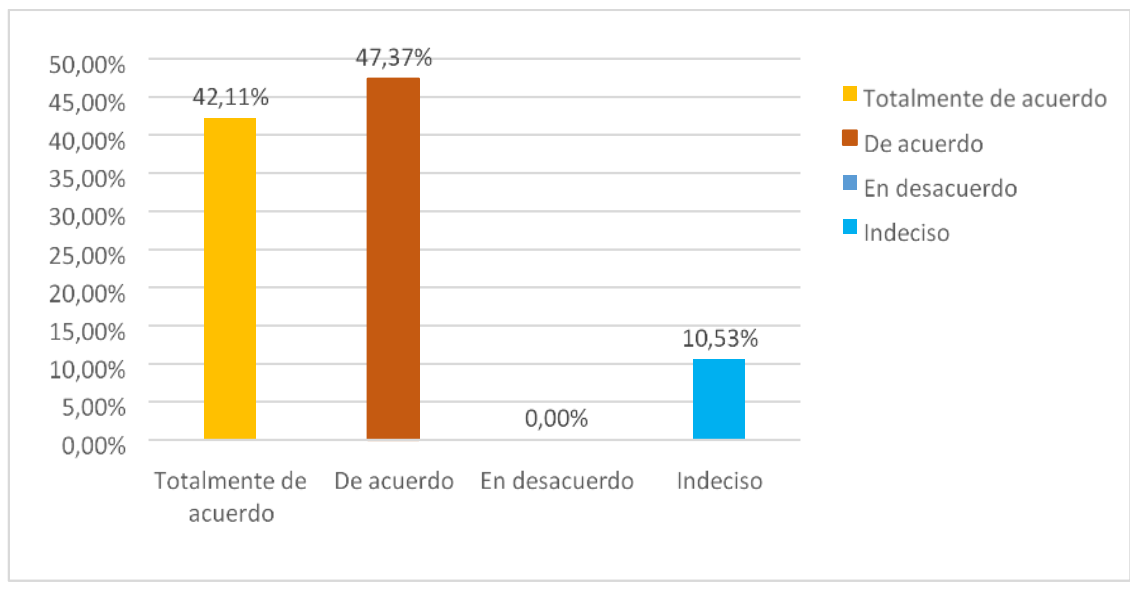

Se evidencia que el $63.16 \%$ de los docentes consideran que es necesaria una capacitación a los estudiantes sobre el uso de las redes sociales orientado a la educación, según como lo menciona (Falla, 2010, pág. 24) Las redes por naturaleza, son formas de interacción social, establecidas como un intercambio muy activo entre personas, comunidades e instituciones. Tengamos en cuenta que el hombre desde sus orígenes ha dado vida a sistemas de comunicación que recaen en la conformación de círculos humanos y hoy en pleno siglo 21, la composición de estos tejidos sociales, se encaminan a ser uno de los retos más relevantes de su historial.

Tabla 4. ¿Cree usted que sea necesaria una capacitación a los estudiantes sobre el uso de las redes sociales orientado a la educación?

\begin{tabular}{ccc}
\hline № & Alternativas & Porcentaje \\
\hline 1 & Totalmente de acuerdo & $21,05 \%$ \\
2 & De acuerdo & $63,16 \%$ \\
3 & Indeciso & $10,53 \%$ \\
4 & En desacuerdo & $5,26 \%$ \\
5 & Totalmente en desacuerdo & $0,00 \%$ \\
\hline
\end{tabular}

\section{Discusión}

Esta investigación centra su importancia de manera fundamental en el uso de las redes sociales y su utilización por parte de los estudiantes y docentes, debido a que 
existe una tendencia creciente en torno a la necesidad de usar las redes sociales como insumos para la acción educativa ya, que se las considera desde diversos puntos de vista un elemento de impulso al desarrollo cognitivo por la facilidad que brindan en cuanto a: las posibilidades de acceso, la interacción permanente y lo llamativo para los estudiantes.

(Freire R. , 2018, pág. 3) menciona que las redes sociales contribuyen en el aprendizaje de los estudiantes dado que, aunque se la realiza en un ambiente informal se puede difundir contenido didáctico e interactivo fomentando así el interés por parte de los estudiantes, tanto para generar contenido o participar en las diversas actividades planteadas. Los estudiantes utilizan las redes sociales para enviar mensajes y comunicarse con sus amigos, familiares $u$ otras personas a las que ya conoce y en una frecuencia casi nula como herramienta de aprendizaje. Los docentes consideran que las mismas permiten al alumno expresarse por sí mismo, entablar relaciones con otros, así como atender a las exigencias propias de su educación. Pienso que las redes sociales deben ser utilizadas por los estudiantes en su proceso de aprendizaje porque estas les permite, centralizar en un único sitio todas las actividades docentes y estudiantes de un centro educativo, aumento del sentimiento de comunidad educativa para estudiantes y profesores debido al efecto de cercanía que producen las redes sociales, aumento en la fluidez y sencillez de la comunicación entre profesores y estudiantes, les facilita la coordinación y trabajo de diversos grupos de aprendizaje mediante la creación de los grupos apropiados.

Para (Hurtado, García, \& Rivera, 2018, pág. 3) La creatividad es la capacidad que tiene una persona para generar ideas o productos originales, novedosos en un contexto social determinado. Es una capacidad inherente al ser humano que debe estimularse para generar las ideas, se debe pasar por un proceso creativo para lograr un estilo de aprendizaje que fomente las capacidades. Los estudiantes y docentes están conscientes de que el uso de las redes sociales les puede aportar significativamente en su creatividad durante el proceso de enseñanza-aprendizaje, pero los 
estudiantes utilizan en un porcentaje mínimo las redes sociales como herramienta pedagógica que les ayude a desarrollar su creatividad. Pienso que la creatividad se puede contemplar en todas las edades, tanto en los docentes como en los estudiantes; pero cuanto antes se comience a cultivar, más posibilidades hay en desarrollar las capacidades de creación, ya que el principal objetivo de la educación es crear hombres capaces de hacer cosas nuevas, siendo las redes sociales unas herramientas más representativas de la Web 2.0, por esto no deben ser obviadas para su estudio, puesto que el eje de todas ellas se adscribe a la interacción y capacidad de responder y comunicar con rapidez y elocuencia.

Como lo menciona (Mujica, 2017, pág. 3) La creatividad en el aprendizaje en línea es sinónimo del uso de dispositivos tecnológicos y pedagógicos implementados con la finalidad de cumplir los objetivos y destrezas planteados a través de la modalidad de Educación virtual acogiéndose al Plan Educativo Aprendamos Juntos en casa que promueve el Ministerio de Educación, el mismo que requiere de responsabilidad y cautela para la utilización de redes sociales, sitios y plataformas Online. La creatividad juega un papel importante en la interrelación de la comunidad educativa por tanto es fundamental la integración de recursos didácticos e interactivos. Aunque los estudiantes utilizan con una frecuencia casi nula las redes sociales para fines pedagógicos, estos mencionan estar de acuerdo con la capacitación a los estudiantes sobre el uso de las redes sociales orientado a la educación, de igual forma los docentes manifiestan estar de acuerdo. Lo que le permitirá a la institución educativa incluir dentro de su propuesta actividades que promuevan el aprendizaje significativo, desarrollando en el estudiante la capacidad de análisis, criticidad y reflexión, con una metodología basada en los soportes tecnológicos. Lo que advierte o avizora el hecho de ver en estos espacios un potencial aliado para la gestión de aula y no solo una amenaza para las prácticas tradicionales. 


\section{Conclusiones}

1. La emergencia sanitaría evidencio debilidades en diferentes sectores del país, el ámbito educativo no fue la excepción, la pandemia covid 19 obligo a las instituciones educativas a impartir clases en línea, lo que desencadeno la dependencia de internet y el uso de herramientas informáticas como recursos educativos digitales. En esta nueva forma de enseñar y aprender incursionaron con gran fuerza el uso de las redes sociales, no solo como medio de comunicación, sino como herramientas digitales para interactuar desde un enfoque colaborativo y cooperativo, entre estudiantes y profesores. De ahí su importancia de usarlas como recurso tecnológico para mejorar en el proceso enseñanza aprendizaje en línea.

Se pudo evidenciar que el uso de las redes sociales por parte de los estudiantes está relacionado a acciones informales, recreativas, comunicativas entre sus pares, mas no con un fin pedagógico ni de aprendizaje. aun siendo estas un medio de comunicación, de información, de formación actual e interactivo tanto de enseñanza como de aprendizaje, ayudándoles a profesores como a alumnos, a familiarizarse con herramientas y aplicaciones. Es menester recalcar, que el uso de las redes sociales contribuye en el aprendizaje de los estudiantes dado que, aunque se la realiza en un ambiente informal se puede difundir contenido didáctico e interactivo fomentando así el interés por parte de los estudiantes.

2. Como se puede evidenciar los estudiantes utilizan muy poco las redes sociales como herramienta pedagógica, como tampoco les dan el mejor uso y no aprovechan todas las facilidades que les brindan estas para el desarrollo de su creatividad en el proceso de enseñanzaaprendizaje. Por lo expuesto es importante que los estudiantes las involucren en sus actividades pedagógicas debido a que en la actualidad las exigencias educativas en línea están obligando a los estudiantes a buscar soluciones basadas en la creatividad, para adaptarse a esta transición o disyuntiva que representa el aprendizaje en línea, para ello se evalúan los procesos sistemáticos obtenidos en el proceso de 
aprendizaje de los estudiantes, tomando en consideración su capacidad creativa, así como los objetivos educativos planteados en el programa escolar, esto se experimenta con el uso de las diferentes herramientas que afianzan la conciencia de la innovación y lo ejercitan en los problemas cotidianos del aprendizaje.

3. Tanto estudiantes como docentes mencionan estar de acuerdo con la capacitación sobre el uso de las redes sociales en el proceso creativo del aprendizaje en línea, ya que son una serie de plataformas digitales que permiten la conexión e interacción entre diversas personas, así como la difusión ilimitada de información, esto ha determinado el interés por el diseño de una propuesta que promueva el uso de ellas, acogiendo diferentes connotaciones que consideren periodos de tiempo para cumplir con objetivos pedagógicos, didácticos, de habilidades, de recursos relacionados con las áreas de estudio, donde se propone que los estudiantes construyan las alternativas en el proceso creativo del aprendizaje en línea que busca proporcionar una posibilidad didáctica enorme para las actividades educativas.

\section{Bibliografía}

Acosta, A., \& Apolo, D. (2018). Reflexiones y Perspectivas sobre los Usos de las Redes Sociales en Educación. Un Estudio de Caso en QuitoEcuador. Información Tecnológica. Obtenido de https://scielo.conicyt.cl/pdf/inf otec/v30n1/0718-0764infotec-30-01-215.pdf

Artero. (2011). El uso de las redes sociales en internet para el incremento del conocimiento y la comunicacion de los estudiantes de la carrera de sociología de la universidad de guayaquil. GuayaquilEcuador: Universidad de Guyayaquil. Obtenido de http://repositorio.ug.edu.ec/bit stream/redug/22160/1/TESIS \%20\%20VICTORIA\%20SAB ANDO\%20Y\%20PATRICIA\% 200CHOA.pdf

Avid, P., \& Bermeo, M. (2018). Las redes sociales en el aprendizaje creativo, Métodos de capacitación sobre el uso de redes sociales. Universidad de Guayaquil. Obtenido de http://repositorio.ug.edu.ec/bit stream/redug/41572/1/BFILO -PD-LP1-17-387A.pdf 
Bohorquéz, \& Rodríguez. (2017). Uso de las redes sociales virtuales y habilidades sociales en adolescentes $y$ jóvenes adultos de lima metropolitana. Lima-Perú: Universidad San Ignacio de Loyola. Obtenido de http://repositorio.usil.edu.pe/b itstream/USIL/2766/1/2017_Ik emiyashiro_Uso-de-lasredes-sociales-virtuales.pdf

Falla. (2010). El uso de las redes sociales en internet para el incremento. Guayaquil: Universidad de Guayaquil. Obtenido de http://repositorio.ug.edu.ec/bit stream/redug/22160/1/TESIS \%20\%20VICTORIA\%20SAB ANDO\%20Y\%20PATRICIA\% 200CHOA.pdf

Freire, Cepeda, \& y Santiago. (2017). Creatividad, microcuentos y redes sociales en el fututo profesorado de Educación Infantil y Primaria. C/ Salud,15 - 5D Madrid, 126. Obtenido de file:///C:/Users/PC/Downloads /5.Creatividadmicrocuentosyr edessocialesenelfututoprofes oradodeEducacionInfantilyPri maria.pdf

Freire, Díaz, \& Vera. (2018). Redes sociales para el aprendizaje significativo: apropiación tecnológica de la web 3.0. Semana de la Ciencia, 3. Obtenido de http://investigacion.utmachala .edu.ec/proceedings/index.ph $\mathrm{p} / \mathrm{utmach} /$ article/download/36 3/309/711

Freire, R. (2018). Redes sociales para el aprendizaje significativo: apropiación tecnológica de la web 3.0. Semana de la Ciencia. Universidad Tecnica de Machala., 3. Obtenido de http://investigacion.utmachala .edu.ec/proceedings/index.ph $\mathrm{p} / \mathrm{utmach} /$ article/download/36 3/309/711

Goméz. (2012). Las redes sociales y su aplicación. Revista Digital Universitaria, Volumen 14 Número 4• ISSN: 1067-6079, 9. Obtenido de http://www.revista.unam.mx/v ol.14/num4/art36/art36.pdf

Hurtado, P., García, M., \& Rivera, D. (2018). Las estrategias de aprendizaje y la creatividad: una relación que favorece el procesamiento de la información. Obtenido de http://www.revistaespacios.co m/a18v39n17/a18v39n17p12. pdf

Kyriacou, S., Negrete, \& René. (2016). El uso de redes sociales en la enseñanza para fomentar el trabajo colaborativo por medio de aprendizaje autónomo. Proceedings-CECORFANMéxico, 7. Obtenido de https://www.ecorfan.org/proc eedings/CDU_XII/TOMO\%20 12_2.pdf 
Meso. (2010). El uso de las redes sociales en internet para el incremento del conocimiento y la comunicación de los estudiantes de la carrera de sociología de la universidad de Guayaquil. Guayaquil: Universidad de Guayaquil. Obtenido de http://repositorio.ug.edu.ec/bit stream/redug/22160/1/TESIS \%20\%20VICTORIA\%20SAB ANDO\%20Y\%20PATRICIA\% 20OCHOA.pdf

Ministerio de Educación. (2020). Plan Educativo Aprendamos Juntos en Cada. Ministerio de Educacion del Ecuador. Obtenido de https://www.gestionderiesgos .gob.ec/wpcontent/uploads/2020/05/Plan -Educativo-AprendamosJuntos-en-casa.pdf

Mujica, R. (2017). La Creatividad en la Educación Virtual. Obtenido de Blog Docentes 2.0:

https://blog.docentes20.com/ 2017/01/la-creatividad-en-laeducacion-virtual/

Muñoz, M., Fragueiro, M., \& Ayuso, M. (2017). La importancia de las redes sociales en el ámbito educativo. Obtenido de

https://dialnet.unirioja.es/desc arga/articulo/4425349.pdf

Organización del Bachillerato Internacional. (2020). Orientación para los colegios sobre la planificación de la continuidad del aprendizaje en línea. Organización del Bachillerato Internacional. Obtenido de https://www.ibo.org/globalass ets/newsassets/coronavirus/onlinelearning-continuity-planninges.pdf

Pérez, Ortíz, \& Flores. (2016). Redes sociales en Educación y propuestas metodológicas para su estudio. Redalyc.org, 18. Obtenido de https://www.redalyc.org/pdf/1 45/14538571008.pdf

Silva, \& Cadena. (2018). Aproximación al uso de las redes sociales en internet desde una revisión teórica. Revista científica. Dominio de las Ciencias, 4. Obtenido de https://www.dominiodelascien cias.com/ojs/index.php/es/arti cle/view/723/html

Villafuerte, J., \& Rodríguez, A. (2019). Redes Sociales y Actitudes Ambientales en Procesos Innovadores de la Formación de Profesionales de Manabí, Ecuador. (pág. 10). Universidad Laica Eloy Alfaro de Manabí. Obtenido de

https://www.researchgate.net/ publication/337567611_Rede s_Sociales_y_Actitudes_Amb ientales_en_Procesos_Innov adores_de_la_formacion_de Profesionales_de_Manabi 